\title{
Existence and Attractivity Results for Volterra Type Nonlinear Perturbed Random Integral Equations
}

\author{
B. D. Karande \\ Department of Mathematics, \\ Maharashtra Udayagiri Mahavidyalaya, \\ Udgir, Maharashtra, India.
}

\author{
S. G. Shete \\ Department of Mathematics, \\ Mahatma Basweshwar College, \\ Latur, Maharashtra, India.
}

\begin{abstract}
In this Paper, we prove an existence and attractivity result for Volterra type nonlinear perturbed random integral equations in separable Banach space under mixed generalized compactness, contraction and caratheodory conditions.
\end{abstract}

Keywords: Banach space, nonlinear random integral equation, existence result, locally attractive solution.

\section{INTRODUCTION}

The coefficients or parameters that have an important role in the natural processes are called random parameters. Hence when we talk about some parameters or coefficients, the random analysis of the random equations is evident. Therefore the random equations have been studied in the literature, since long time, by various Mathematicians all over the world. Thus the study of a natural or physical phenomenon with the help of random models or equations forms an important branch of the analysis. Random fixed point theorems are the stochastic generalizations of the classical or deterministic fixed point theorems in abstract spaces and are useful in the study of nonlinear random equations for proving the existence theorems $[5,6,7,9,10]$. In this paper we study the existence of locally attractive solution for Volterra type nonlinear perturbed random integral equations in separable Banach space employing random versions of the Krasnoselskii fixed point theorem of Krasnoselskii and B. C. Dhage in the form of Schaefer fixed point theory.

Let $\mathbb{R}$ denotes the real numbers and $\mathbb{R}_{+}$be a set of nonnegative real numbers, that is $\mathbb{R}_{+}=(0, \infty) \subset \mathbb{R}$ and let $\mathrm{X}$ is a separable Banach space with the norm $\|\cdot\|$.

Now consider the nonlinear Volterra type perturbed random integral equation of the type

$$
x(t, \omega)=h(t, x(t, \omega))+\int_{0}^{t} k(t, \tau, \omega) f(\tau, x(\tau, \omega)) d \tau
$$

for every $t \geq 0$,

where the random perturbed term $h(t, x(t, \omega))$ is a mapping from $\mathbb{R}_{+} \times \mathbb{R} \rightarrow \mathbb{R}$. $\omega$ is the point of $\Omega$ and $x(t, \omega)$ is an unknown random variable for eacht $\geq 0$. The kernel $k(t, \tau, \omega)$ is a mapping from $\mathbb{R}_{+} \times \mathbb{R}_{+} \times \Omega \rightarrow \mathbb{R}$ and is defined for $0 \leq \tau \leq t \leq \infty$ and $\omega \in \Omega$, the kernel $k(t, \tau, \omega)$ is an essentially bounded function with respect to measure $\mu$ for every $t \& \tau$ such that $0 \leq \tau \leq t \leq \infty$. The function $f(\tau, x(\tau, \omega))$ is a mapping from $\mathbb{R}_{+} \times \mathbb{R} \rightarrow \mathbb{R}$ for $t \geq 0$. i.e. the function $f$ is an scalar valued function for $\tau \geq 0$.

The measurable function $x: \Omega \rightarrow C\left(\mathbb{R}_{+}, \mathbb{R}\right)$ is said to be the random solution of equation (1.1) if for every $t \in \mathbb{R}_{+}$, it satisfy the nonlinear Volterra type perturbed random integral equation (1.1). where the space $C\left(\mathbb{R}_{+}, \mathbb{R}\right)$ denotes the space of all continuous real valued functions on $\mathbb{R}_{+}$. 


\section{Preliminaries}

In this section, we present here some notations, definitions and preliminary facts that will be used in the proofs of our main results.

Definition 2.1 A mapping $\mathrm{T}: \Omega \times \mathrm{X} \rightarrow \mathrm{X}$ is called a random operator, if $T(\omega, x)$ is measurable for each $x \in \mathrm{X}$ and this random operator is generally denoted as $T(\omega) x$ and it expressed as $T(\omega) x=$ $T(\omega, x)$.

Definition 2.2 A random variable $\eta: \Omega \rightarrow X$ is called a random fixed point of a random operator $T(\omega): \Omega \times X \rightarrow X$, if $T(\omega) \eta(\omega)=n(\omega)$ for every $\omega \in \Omega$.

Definition 2.3 A random operator $\mathrm{T}(\omega): \Omega \times X \rightarrow \mathrm{X}$ is called a totally bounded, if for any bounded set $D$ in $X, T(\omega)(D)$ is totally bounded subset of $X$ for every $\omega \in \Omega$.

Definition 2.4 A random operator $\mathrm{T}(\omega)$ is called a completely continuous on Banach space $\mathrm{X}$, if it is totally bounded and continuous random operator on $\mathrm{X}$.

Definition 2.5 An random operator $T(\omega): \Omega \times X \rightarrow X$ is called compact random operator, if $\overline{T(\omega)(X)}$ is compact subset of $X$ where $\overline{T(\omega)(X)}$ is the closure of $T(\omega)(X)$ in $X$.

Definition 2.6 A random operator $T(\omega): \Omega \times X \rightarrow X$ is called a $D$-Lipschitzician, if there exist a nondecreasing continuous function $\varphi: \Omega \times \mathbb{R}_{+} \rightarrow \mathbb{R}_{+}$such that for every $\omega \in \Omega$

$$
\|T(\omega) x-T(\omega) y\| \leq \varphi_{\omega}(\|x-y\|)
$$

for all $x, y \in X$, where $\varphi_{\omega}(r)=\varphi(\omega, r)$ with $\varphi(\omega, 0)=0$. Here the some special case $\varphi_{\omega}(r)=\alpha(\omega) r_{x}$ for $\alpha(\omega)>0$ for every $\omega \in \Omega$ then the random operator $T(\omega)$ is called a Lipschtzician with Lipschitz constant $\alpha(\omega)$, for $\omega \in \Omega$. In particular for each $\in \Omega, \alpha(\omega)<1$ then random operator $T(\omega)$ is known as a contraction with contraction constant $\alpha(\omega)$. and if $\varphi_{\omega}(r)<r$, for $r>0$ and for every $\omega \in \Omega$ then $T(\omega)$ is known as a nonlinear contraction.

Definition 2.7 (Dugundji and Granas[8]):An operator A on a Banach space $\mathrm{X}$ into itself is called Compact, if for any bounded subset $\mathrm{S}$ of $\mathrm{X}, \mathrm{A}(\mathrm{S})$ is a relatively compact subset of $\mathrm{X}$. If $\mathrm{A}$ is continuous and compact, then it is called completely continuous on $\mathrm{X}$.

Theorem 2.1 (Arzela-Ascoli theorem): If every uniformly bounded and equi-continuous sequence $\left\{f_{n}\right\}$ of functions in $C\left(\mathbb{R}_{+}, \mathbb{R}\right)$, then it has a convergent subsequence.

Theorem 2.2 (Dominated convergence theorem) Let $\left\{f_{n}\right\}$ be a sequence of increasing nonnegative measurable functions on a measurable subset $\mathrm{M}$ of $\mathbb{R}$, if for all $x \in M,\left\|f_{n}(x)\right\| \leq h(x)$ for some measurable function $h$ with $\lim _{n \rightarrow-s} f_{n}(x)=f(x)$ for almost every $x \in M$, then

$$
\lim _{n \rightarrow \infty} \int_{M} f_{n} d \mu=\int_{M} \lim _{n \rightarrow \infty} f_{n} d \mu=\int_{M} f d \mu
$$

Theorem 2.3 (Monotone convergence theorem):Let $\left\{f_{n}\right\}$ be a sequence of increasing nonnegative measurable functions withlim $m_{n \rightarrow=s} f_{n}(x)=f(x)$, then the function $f$ is a measurable function and

$$
\int_{M} \lim _{n \rightarrow \infty} f_{n} d \mu=\int_{M} f d \mu
$$

Now we shall seek the existence of the solution to the nonlinear Volterra type random integral equation of the type in the space BM $\left(\mathbb{R}_{+}, \mathbb{R}\right)$ be the space of all bounded and measurable real valued functions on $\mathbb{R}_{+}$with the norm is defined as

$$
\|x\|_{B N}=\max _{t \in R t}\|x(t)\|
$$


under some suitable conditions.

Let $\mathrm{X}=\mathrm{BM}\left(\mathbb{R}_{+}, \mathbb{R}\right)$ be the space of all bounded and measurable real valued functions on $\mathbb{R}_{+}$and $\Omega$ be a nonempty subset of $X$. Let $\varphi: \Omega \times X \rightarrow X$ be a mapping and consider the following random equation

$$
\varphi(\omega) x(t)=x(t, \omega)
$$

for $\mathrm{t} \geq 0$, and $\omega \in \Omega$.

Below we give different characterizations of the solutions for operator equation (2.2) on $\mathbb{R}_{+}$, we need the following definitions.

Definition 2.8: B. C. Dhage [1] The random equation (2.2) has said to be have locally attractive random solution on $\mathbb{R}_{+}$, if there exists a closed ball $\overline{B_{r}}\left(x_{1}\right)$ in the space $\operatorname{CB}\left(\mathbb{R}_{+}, \mathbb{R}\right)$ for some $x_{1} \in \mathrm{CB}\left(\mathbb{R}_{+}, \mathbb{R}\right)$ and for any real number $r \geq 0$ such that for any random solution $x(t, \omega)$ and $y(t, \omega)$ of the random equation $(2.2)$ belonging to $\overline{B_{r}}\left(x_{1}\right) \cap C$, we have

$$
\lim _{n \rightarrow \infty}(x(t, \omega)-y(t, \omega))=0
$$

for every $\omega \in \Omega$. Here in this case when the limit of equation $(2.3)$ is uniform with respect to the set $\overline{B_{r}}\left(x_{1}\right) \cap C$. i.e. every $\varepsilon>0$ then there exists a $\mathrm{T}>0$ such that for every $\mathrm{t} \geq \mathrm{T}$,

$$
\llbracket x(t, \omega)-y(t, \omega) \rrbracket \leq \varepsilon
$$

for every $\omega \in \Omega$ and $x_{y} y \in \overline{B_{r}}\left(x_{1}\right) \cap C$ being the random solution of equation $(2.2)$, then we will say that the random solution is uniformly locally attractive on $\mathbb{R}_{+}$.

Concepts of the locally attractivity of solutions were introduced in [1, 6, and 7].

Now we give random versions of the Krasnoselskii fixed point theorem Krasnoselskii [9] and B. C. Dhage [4] in the form of Schaefer fixed point theory [2] which is the main tool in the existence theorem of solutions to the nonlinear Volterra type perturbed random integral equation (1.1).

Theorem 2.4: B. C. Dhage [2] Let $X$ be a separable Banach space, $T(\omega), S(\omega): X \rightarrow X$ be two random operators satisfying for every $\omega \in \Omega_{\text {, }}$

i) $\mathrm{S}(\omega)$ is nonlinear contraction,

ii) $T(\omega)$ is completely continuous,

iii) The set $\eta=\{x \in X / S(\omega) x+T(\omega) x=\alpha(\omega) x\}$ is bounded, for any measurable function $\alpha: \Omega \rightarrow \mathbb{R}^{+}$with $\alpha(\omega)>1$.

Then the random equation $S(\omega) x+T(\omega) x=x$ has a random solution.

An interesting corollary to Theorem (2.4) in its applicable form is

Corollary 2.1: B. C. Dhage [2] Let $X$ be a separable Banach space and let $T, S: Q \times X \rightarrow X$ be two random operators satisfying for every $\omega \in \Omega_{\text {, }}$

i) $\mathrm{S}(\omega)$ is contraction,

ii) $T(\omega)$ is completely continuous,

iii) The set $\eta=\{x \in X / S(\omega) x+T(\omega) x=\alpha(\omega) x\}$ is bounded, for any measurable function $\alpha: \Omega \rightarrow \mathbb{R}^{+}$with $\alpha(\omega)>1_{x}$ then the random equation $S(\omega) x+T(\omega) x=x$ has a random solution. 


\section{EXISTENCE RESULT}

Let $\mathbb{R}$ be the real line and let $B\left(\mathbb{R}_{+}, \mathbb{R}\right)$ and $M\left(\mathbb{R}_{+}, \mathbb{R}\right)$ denotes the space of all bounded and measurable real valued functions on $\mathbb{R}_{+}$respectively, and the space $B M\left(\mathbb{R}_{+}, \mathbb{R}\right)$ of all bounded and measurable real valued functions on $\mathbb{R}_{+}$with the norm is defined as

$$
\|x\|_{B N}=\max _{t \in R+}\|x(t)\|
$$

Then clearly, the space $B M\left(\mathbb{R}_{+}, \mathbb{R}\right)$ is Banach space with respect to this maximum norm defined above and also the space $B M\left(\mathbb{R}_{+}, \mathbb{R}\right)$ is a separable Banach space with respect to this maximum norm defined above. $C\left(\mathbb{R}_{+}, \mathbb{R}\right)$ denotes the space of all continuous real valued functions on $\mathbb{R}_{+}$and we get the relation between them as $\mathrm{C}\left(\mathbb{R}_{+}, \mathbb{R}\right) \subset B M\left(\mathbb{R}_{+}, \mathbb{R}\right) \subset M\left(\mathbb{R}_{+}, \mathbb{R}\right)$. (B. C. Dhage [2])

We define the norm in $C\left(\mathbb{R}_{+}, \mathbb{R}\right)$ which is denoted by $\|x\|_{0}$ and defined as,

$$
\|x\|_{\mathbb{e}}=\sup \|x(t)\| \text { for } t \in \mathbb{R}_{+} .
$$

The space $\mathrm{L}^{1}\left(\mathbb{R}_{+}, \mathbb{R}\right)$ denotes the space of all Lebesgue integral real valued function on $\mathbb{R}_{+}$with respective to the $\|.\|_{L^{1}}$ defined by

$$
\|x\|_{L^{\mathbb{1}}}=\int_{t_{1}}^{\tau_{1}} \llbracket x(s) \| d s
$$

We need some following definitions,

Definition 3.1: A function $\beta: \mathbb{R}_{+} \times \mathbb{R} \times \Omega \rightarrow \mathbb{R}$ is said to be random caratheodory function, if

i) The map $t \rightarrow \beta(t, x)$ is jointly measurable for all $x \in \mathbb{R}$.

ii) $x \rightarrow \beta(t, x)$ is continuous for almost every $t \in \mathbb{R}_{+}$.

Definition 3.2: B. C. Dhage [2] A random Caratheodory function $\beta: \mathbb{R}_{+} \times \mathbb{R} \times \Omega \rightarrow \mathbb{R}$ is said to be random $\mathrm{L}^{1}$-caratheodory function, if

i) For each real number $r>0$, there is measurable and bounded function $q_{r} \in L^{1}\left(\mathbb{R}_{+}, \mathbb{R}\right)$ such that $\|\beta(t, x(t, \omega))\| \leq q_{r}(t, \omega)$ for all $t \in \mathbb{R}_{+}$and $x \in \mathbb{R}$ with $\|x\| \leq r$.

Now we consider the following some hypothesis,

$\left(\mathrm{H}_{0}\right)$ The function $h: \mathbb{R}_{+} \times \mathbb{R} \rightarrow \mathbb{R}$ is measurable,

$\left(\mathrm{H}_{1}\right)$ The function $k: \mathbb{R}_{+} \times \mathbb{R} \times \Omega \rightarrow \mathbb{R}$ are continuous and bounded with bound $\mathrm{K}(\omega)$,

$\left(\mathrm{H}_{2}\right)$ The function $\omega \rightarrow f(t, x(t, \omega))$ is measurable for all $t \in \mathbb{R}_{+}$

$\left(\mathrm{H}_{3}\right)$ The function $h: \mathbb{R}_{+} \times \mathbb{R} \rightarrow \mathbb{R}-\{0\}$ is a continuous function and there exist a bounded function $\alpha: \mathbb{R}_{+} \rightarrow \mathbb{R}_{+}$such that

$$
|h(t, x(t, \omega))-h(t, y(t, \omega))| \leq \alpha(t)|x(t, \omega)-y(t, \omega)|
$$

for all $t \in \mathbb{R}_{+}$, with $\|\alpha(t)\|<1$ and $\lim _{t \rightarrow \infty} h(t, \omega)=0, \lim _{t \rightarrow \infty} \alpha(t)=0$,

$\left(\mathrm{H}_{4}\right)$ The function $f: \mathbb{R}_{+} \times \mathbb{R} \rightarrow \mathbb{R}$ is an random $\mathrm{L}^{1}-$ caratheodory function,

$\left(\mathrm{H}_{5}\right)$ The uniform continuous function $v: \mathbb{R}_{+} \rightarrow \mathbb{R}$ defined by the formulas $v(t, \omega)=\int_{0}^{t} q_{r}(\tau, \omega) d \tau$ is bounded with bound $\left\|q_{r}\right\|_{L^{1}}$ on $\mathbb{R}_{+}$and vanish at infinity, that is, $\lim _{t \rightarrow \infty} v(t, \omega)=0$.

Remark 3.1: Note that, if the hypothesis $\left(\mathrm{H}_{4}\right)-\left(\mathrm{H}_{5}\right)$ hold, then there exist constant $\mathrm{K}_{1}>0$ such that $K_{1}=\sup \left\{K(\omega) v(t, \omega) \backslash t \in \mathbb{R}_{+}\right.$and $\left.\omega \in \Omega\right\}$. 
Theorem 3.1: Suppose that the hypothesis $\left(\mathrm{H}_{0}\right)$ to $\left(\mathrm{H}_{5}\right)$ holds, further if there exist a real number $r>0$ such that $\left\|h_{k}\right\|+\|K(\omega)\|\left\|q_{r}\right\|_{L^{\mathbb{1}}}=r$, then the nonlinear Volterra type perturbed random integral equation (1.1) has solution. And moreover, random solutions are locally attractive on $\mathbb{R}_{+}$.

Proof: Let $\mathrm{X}=\mathrm{BM}\left(\mathbb{R}_{+}, \mathbb{R}\right)$ be a separable Banach space. Now we define the two operators $\mathrm{A}$ and $\mathrm{B}$ on $\mathrm{X}$, by

$$
\begin{aligned}
& A(\omega) x(t)=h(t, x(t, \omega)) \\
& B(\omega) x(t)=\int_{0}^{t} k(t, \tau, \omega) f(\tau, x(\tau, \omega)) d \tau
\end{aligned}
$$

Then the nonlinear Volterra type random perturbed integral equation (1.1) is equivalent to the operator equation

$$
A(\omega) x(t)+B(\omega) x(t)=x(t, \omega)
$$

for all $t \in \mathbb{R}_{+}$, hence the problem of the existence of solution to the nonlinear Volterra type random perturbed integral equation (1.1) is to just reduced to the finding the solution to the operator equation

$$
A(\omega) x(t)+B(\omega) x(t)=x(t, \omega) \text { in } \mathrm{X} .
$$

Step I: First, we show that $A(\omega)$ and $B(\omega)$ are random operators on $\mathrm{X}$.

Since by the hypothesis $\left(\mathrm{H}_{0}\right),\left(\mathrm{H}_{1}\right)$ and $\left(\mathrm{H}_{2}\right)$, as the function $\omega \rightarrow h(t, x(t, \omega)), \omega \rightarrow k(t, \tau, \omega)$ and $\omega \rightarrow f(\tau, x(\tau, \omega))$ are measurable on $\mathrm{X}$, and the space $\mathrm{X}$ is a separable Banach space then the function $\omega \rightarrow k(t, \tau, \omega) f(\tau, x(\tau, \omega))$ is measurable for all $t, \tau \in \mathbb{R}_{+}$, and also the integral

$$
\int_{0}^{\tau} k(t, \tau, \omega) f(\tau, x(\tau, \omega)) d \tau
$$

is the limit of a finite sum of measurable functions, so that the function

$$
\omega \rightarrow \int_{0}^{t} k(t, \tau, \omega) f(\tau, x(\tau, \omega)) d \tau
$$

is measurable.

Hence the operator $A(\omega)$ and $B(\omega)$ are random operators on $\mathrm{X}$.

Now we show that the random operators $A(\omega)$ and $B(\omega)$ are satisfying all the conditions of corollary 2.1 .

Step II: Now we show that the random operator $A(\omega)$ is a contraction on $X$.

Let $x, y \in \mathrm{X}$ be any arbitrary elements, then by the hypothesis $\left(\mathrm{H}_{3}\right)$, we get,

$$
\begin{gathered}
\|A(\omega) x(t)-A(\omega) y(t)\|=|h(t, x(t, \omega))-h(t, y(t, \omega))| \\
\leq \alpha(t)\|x(t, \omega)-y(t, \omega)\|
\end{gathered}
$$

Taking maximum all over $\mathrm{t}$, we get,

$$
\begin{aligned}
\|A(\omega) x(t)-A(\omega) y(t)\| & \leq\|\alpha(t)\|\|x(t, \omega)-y(t, \omega)\| \\
& \leq\|\alpha(t)\|\|x-y\| \\
\|A(\omega) x(t)-A(\omega) y(t)\| & \leq\|\alpha(t)\|\|x-y\|
\end{aligned}
$$

provided that $\|\alpha(t)\|<1$.

Thus the random operator $A(\omega)$ is a contraction on $\mathrm{X}$. 
Step III: The operator $B(\omega)$ is continuous operator on X.

Let $\mathrm{Y}$ be a bounded subset of $\mathrm{X}$, then there is real number $r>0$ such that $\|x\| \leq r$ for all $y \in Y$, let $\left\{y_{n}\right\}$ be a convergent sequence of points in $\mathrm{Y}$ converging to the point $\mathrm{y} \in \mathrm{Y}$. Then it is enough to prove that $\lim _{n \rightarrow \infty} B(\omega) y_{n}(t)=B(\omega) y(t), t \in \mathbb{R}_{+}$, By the Lebesgue dominated converging theorem, we obtain,

$$
\begin{aligned}
& \lim _{n \rightarrow \infty} B(\omega) y_{n}(t)=\lim _{n \rightarrow \infty} \int_{0}^{t} k(t, \tau, \omega) f\left(\tau, y_{n}(\tau, \omega)\right) d \tau \\
& \leq \int_{0}^{t} k(t, \tau, \omega) \lim _{n \rightarrow \infty} f\left(\tau, y_{n}(\tau, \omega)\right) d \tau \\
& \leq \int_{0}^{t} k(t, \tau, \omega) f(\tau, y(\tau, \omega)) d \tau \\
&=\mathrm{B}(\omega) y(t)
\end{aligned}
$$

for every $t \in \mathbb{R}_{+}, \omega \in \Omega$.

This proves that the random operator $\mathrm{B}(\omega)$ is continuous operator on $\mathrm{X}$.

Step IV: The operator $\mathrm{B}(\omega)$ is compact operator on $\mathrm{X}$.

Firstly, we show that $\{\mathrm{B}(\omega) \mathrm{Y}\}$ is uniformly bounded and equicontinuous set in $\mathrm{X}$ for each $\omega \in \Omega$.

Let $x$ be arbitrary element in $\mathrm{Y}$ then by hypothesis $\left(\mathrm{H}_{4}\right)$ the function $f(t, x(t, \omega))$ is $\mathrm{L}^{1}$-caratheodory function, we have

$$
\begin{aligned}
\|B(\omega) x(t)\| & =\left|\int_{0}^{t} k(t, \tau, \omega) f(\tau, x(\tau, \omega)) d \tau\right| \\
& \leq \int_{0}^{t}|k(t, \tau, \omega) f(\tau, x(\tau, \omega))| d \tau \\
& \leq \int_{0}^{t}\|k(t, \tau, \omega)\| f(\tau, x(\tau, \omega)) \mid d \tau \\
& \leq K(\omega) \int_{0}^{t}|f(\tau, x(\tau, \omega))| d \tau \\
& \leq K(\omega) \int_{0}^{t}\left|q_{r}(\tau)\right| d \tau \\
\|B(\omega) x(t)\| \leq K(\omega) v(t, \omega) &
\end{aligned}
$$

Taking suprimum all over $\mathrm{t}$, we obtain

$\|B(\omega) x(t)\| \leq K_{1}$

for all $x(t) \in Y$, this shows that the $\{B(\omega) Y\}$ is a uniformly bounded subset in $X$ for each $\omega \in \Omega$.

Secondly, we show that $B(\omega) Y$ is an equicontinuous set in $X$. 
Let $x \in Y$ be arbitrary, then for anys, $t \in \mathbb{R}_{+}$with $0<s<t<\infty$ and for some $\omega \in \Omega$, equation (3.2) implies that

$$
\begin{aligned}
& \| B(\omega) x(t)-B(\omega) x(s) \rrbracket= \\
& =\left|\int_{0}^{\tau} k(t, \tau, \omega) f(\tau, x(\tau, \omega)) d \tau-\int_{0}^{s} k(s, \tau, \omega) f(\tau, x(\tau, \omega)) d \tau\right| \\
& \leq\left|\int_{0}^{\Omega} k(t, \tau, \omega) f(\tau, x(\tau, \omega)) d \tau+\int_{\Omega}^{\tau} k(t, \tau, \omega) f(\tau, x(\tau, \omega)) d \tau-\int_{0}^{s} k(s, \tau, \omega) f(\tau, x(\tau, \omega)) d \tau\right| \\
& \leq\left|\int_{0}^{s} k(t, \tau, \omega) f(\tau, x(\tau, \omega)) d \tau-\int_{0}^{s} k(s, \tau, \omega) f(\tau, x(\tau, \omega)) d \tau\right|+\left|\int_{\Omega}^{\tau} k(t, \tau, \omega) f(\tau, x(\tau, \omega)) d \tau\right| \\
& \leq\left|\int_{0}^{s}(k(t, \tau, \omega)-k(s, \tau, \omega)) f(\tau, x(\tau, \omega)) d \tau+\int_{s}^{t} k(t, \tau, \omega) f(\tau, x(\tau, \omega)) d \tau\right| \\
& \leq \int_{0}^{s}\|k(t, \tau, \omega)-k(s, \tau, \omega)\||f(\tau, x(\tau, \omega))| d \tau+\int_{\Xi}^{t}\|k(t, \tau, \omega)\| f(\tau, x(\tau, \omega)) \mid d \tau \\
& \leq \int_{0}^{\Xi}\|k(t, \tau, \omega)-k(s, \tau, \omega)\||f(\tau, x(\tau, \omega))| d \tau+K(\omega) \int_{\mathbb{Z}}^{\tau}|f(\tau, x(\tau, \omega))| d \tau \\
& \leq \int_{0}^{\pi}\|k(t, \tau, \omega)-k(s, \tau, \omega)\|\left\|q_{r}(\tau, \omega)\right\| d \tau+K(\omega) \int_{\mathbb{a}}^{t}\left\|q_{r}(\tau, \omega)\right\| d \tau \\
& \leq \int_{0}^{\pi}\|k(t, \tau, \omega)-k(s, \tau, \omega)\|\left\|q_{r}(\tau, \omega)\right\| d \tau+K(\omega)\|v(t, \omega)-v(s, \omega)\|
\end{aligned}
$$

for all $\omega \in \Omega$.

Since $v$ is uniformly continuous function, it follows that $\|B(\omega) x(t)-B(\omega) x(s)\| \rightarrow 0$ as $t \rightarrow s$ for all $x \in \mathrm{Y}$ and $\omega \in \Omega$. Therefore $\{\mathrm{B}(\omega) \mathrm{Y}\}$ is an equicontinuous set in $\mathrm{X}$ for all $s, t \in \mathbb{R}_{+}$.

As $\{\mathrm{B}(\omega) \mathrm{Y}\}$ is uniformly bounded and equicontinuous subset in $\mathrm{X}$, it is compact in $\mathrm{X}$ by Arzela Ascoli theorem for each $\omega \in \Omega$. As a consequence, $\mathrm{B}(\omega)$ is compact and continuous operator on $\mathrm{X}$.

Thus B is completely continuous on $\mathrm{X}$.

Step V: Now for any solution $x$ to $A(\omega) x+B(\omega) x=\alpha(\omega) x$ for $\alpha(\omega)>1$, then it has

$$
\begin{aligned}
\|x(t, \omega)\|=\mid h(t, x(t, \omega)) & +\int_{0}^{t} k(t, \tau, \omega) f(\tau, x(\tau, \omega)) d \tau \mid \\
& \leq|h(t, x(t, \omega))|+\left|\int_{0}^{t} k(t, \tau, \omega) f(\tau, x(\tau, \omega)) d \tau\right| \\
& \leq|h(t, x(t, \omega))|+\int_{0}^{t}|k(t, \tau, \omega) f(\tau, x(\tau, \omega))| d \tau \\
& \leq|h(t, x(t, \omega))|+\int_{0}^{t}|k(t, \tau, \omega)||f(\tau, x(\tau, \omega))| d \tau
\end{aligned}
$$




$$
\begin{aligned}
& \leq|h(t, x(t, \omega))|+K(\omega) \int_{0}^{t}|f(\tau, x(\tau, \omega))| d \tau \\
& \leq|h(t, x(t, \omega))|+K(\omega) \int_{0}^{t}\left\|q_{r}(\tau, \omega)\right\| d \tau \\
& \leq h_{k}(t, \omega)+K(\omega)\left\|q_{r}\right\|_{L^{\mathbb{1}}}
\end{aligned}
$$

Taking maximum on $\mathrm{t}$, we get

$$
\|x(t, \omega)\| \leq\left\|h_{k}\right\|+\|K(\omega)\|\left\|q_{r}\right\|_{L^{1}}=r
$$

$\|x(t, \omega)\| \leq r$ for all $\omega \in \Omega$ and for all $t \in \mathbb{R}_{+}$. Thus all the condition of corollary 2.1 are satisfied and hence the application this corollary 2.1 yields that the random operator equation (3.3) and consequently the nonlinear Volterra type perturbed random integral equation (1.1) has a random solution in $\mathrm{X}$.

Step VI: Finally, we show the locally attractive of the solutions for (1.1). Let $x$ and $y$ be any two solutions of the nonlinear Volterra type perturbed random integral equation (1.1) in X defined on $\mathbb{R}_{+}$. Then we have,

$$
\begin{aligned}
& \|x(t, \omega)-y(t, \omega)\|=\mid \begin{array}{c}
h(t, x(t, \omega))+\int_{0}^{t} k(t, \tau, \omega) f(\tau, x(\tau, \omega)) d \tau-(h(t, y(t, \omega)) \\
\left.+\int_{0}^{t} k(t, \tau, \omega) f(\tau, y(\tau, \omega)) d \tau\right)
\end{array} \\
& =\left|\begin{array}{c}
h(t, x(t, \omega))+\int_{0}^{t} k(t, \tau, \omega) f(\tau, x(\tau, \omega)) d \tau-h(t, y(t, \omega)) \\
-\int_{0}^{t} k(t, \tau, \omega) f(\tau, y(\tau, \omega)) d \tau
\end{array}\right| \\
& \leq\left|h(t, x(t, \omega))-h(t, y(t, \omega))+\int_{0}^{t} k(t, \tau, \omega) f(\tau, x(\tau, \omega)) d \tau-\int_{0}^{t} k(t, \tau, \omega) f(\tau, y(\tau, \omega)) d \tau\right| \\
& \leq|h(t, x(t, \omega))-h(t, y(t, \omega))|+\left|\int_{0}^{t} k(t, \tau, \omega) f(\tau, x(\tau, \omega)) d \tau-\int_{0}^{t} k(t, \tau, \omega) f(\tau, y(\tau, \omega)) d \tau\right| \\
& \leq|h(t, x(t, \omega))-h(t, y(t, \omega))|+\left|\int_{0}^{t} k(t, \tau, \omega) f(\tau, x(\tau, \omega)) d \tau\right|+\left|\int_{0}^{t} k(t, \tau, \omega) f(\tau, y(\tau, \omega)) d \tau\right| \\
& \leq \alpha(t)|x(t, \omega)-y(t, \omega)|+\int_{0}^{t}|k(t, \tau, \omega) f(\tau, x(\tau, \omega))| d \tau+\int_{0}^{t}|k(t, \tau, \omega) f(\tau, y(\tau, \omega))| d \tau \\
& \leq \alpha(t)\|x(t, \omega)-y(t, \omega)\|+\int_{0}^{t}\|k(t, \tau, \omega)\||f(\tau, x(\tau, \omega))| d \tau+\int_{0}^{t}\|k(t, \tau, \omega)\||f(\tau, y(\tau, \omega))| d \tau \\
& \leq \alpha(t)\|x(t, \omega)-y(t, \omega)\|+K(\omega) \int_{0}^{ \pm}|f(\tau, x(\tau, \omega))| d \tau+K(\omega) \int_{0}^{ \pm}|f(\tau, y(\tau, \omega))| d \tau
\end{aligned}
$$




$$
\begin{aligned}
& \leq \alpha(t)\|x(t, \omega)-y(t, \omega)\|+K(\omega) \int_{0}^{t} q_{r}(\tau, \omega) d \tau+K(\omega) \int_{0}^{t} q_{r}(\tau, \omega) d \tau \\
& \leq \alpha(t)\|x(t, \omega)-y(t, \omega)\|+2 K(\omega) \int_{0}^{t} q_{r}(\tau, \omega) d \tau
\end{aligned}
$$

Taking the limit superior in the above inequality as $t$ tends to $\infty$ yield

$$
\begin{aligned}
& \lim _{t \rightarrow \infty} \sup \|x(t, \omega)-y(t, \omega)\| \leq \lim _{t \rightarrow \infty} \sup \alpha(t)\|x(t, \omega)-y(t, \omega)\|+\lim _{t \rightarrow \infty} \sup 2 K(\omega) \int_{0}^{t} q_{r}(\tau, \omega) d \tau \\
& \lim _{t \rightarrow \infty} \sup \|x(t, \omega)-y(t, \omega)\| \leq \lim _{t \rightarrow \infty} \sup \alpha(t)\|x(t, \omega)-y(t, \omega)\|+2 K(\omega) \lim _{t \rightarrow \infty} \sup v(t, \omega) \\
& . \quad \lim _{t \rightarrow \infty} \sup \|x(t, \omega)-y(t, \omega)\|=0, \text { since } \lim _{t \rightarrow \infty} \sup v(t, \omega)=0 \text { and } \lim _{t \rightarrow \infty} \sup \alpha(t)=0 .
\end{aligned}
$$

Then from above inequality it follows that $\|x(t, \omega)-y(t, \omega)\| \leq \epsilon$ for all $t \in \mathbb{R}_{+}$.

This completes the proof.

\section{REFERENCES}

[1] B. C. Dhage and S. S. Bellale, Local asymptotic stability for nonlinear quadratic functional integral equations, Electronic journal of qualitative theory of differential equations, vol-10, pg. no.1-13, (2008).

[2] B. C. Dhage, A random version of schaefer's fixed point theorem with applications to functional random integral equations, tamkang journal of mathematics, volume 35, number 3, autumn 2004 , pg. no. 197-205, (2004).

[3] B. C. Dhage, Monotone iterative technique for Caratheodory theory of nonlinear functional random integral equations, Tamkang J. Math. 33 (4), pg. no. 341-351, (2002).

[4] B. C. Dhage, On a-condensing mappings in Banach algebras, The Math. Student 64, pg. no.146152, (1994).

[5] B. C. Dhage, Remarks on Two Fixed-Point Theorems Involving the Sum and the Product of Two Operators, int. journal of Computers and Mathematics with Applications 46, pg. no.1779-1785, (2003).

[6] B. D. Karande, S. V. Kendre and R. N. Ingle, Attractivity Results for Fractional Order Nonlinear Random Integral Equation, International Journal of Scientific and Innovative Mathematical Research, Volume-3, Special Issue-2, 546-557, (July 2015).

[7] B. D. Karande, Global attractively of solutions for a nonlinear functional integral equation of fractional order in Banach Space, AIP Conference Proceeding-American Institute of Physics "10th international Conference on Mathematical Problems in Engineering, Aerospace and Sciences"1637, 469-478, (2014).

[8] Dugunji. J. and Granas A., Fixed point theory, in Monographie Math., Warsaw, (1982).

[9] M. A. Krasnosel'skii, Topological Methods in the Theory of Nonlinear Integral Equations, Pergamon Press, 1964.

[10] S. Itoh, Random fixed-point theorems with an application to random differential equations in Banach spaces, Journal. Math. Anal. Appl. 67, pg. no.261-273, (1979). 


\section{AUTHORS' BIOGRAPHY}

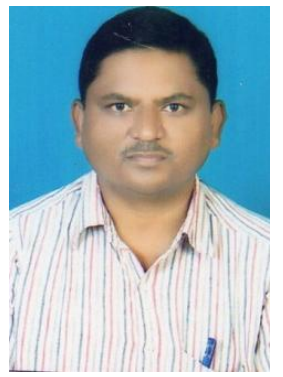

Dr. B. D. Karande, was born on 01.07.1974.He was awarded his Ph.D. in the area of nonlinear differential and integral equations in Banach algebras from S.R.T.M.U. Nanded in the year 2008. Presently he is working as Head, Department of Mathematics, Maharashtra Udayagiri College, Udgir, Dist. Latur, Maharashtra, since 2008. He started his career as lecturer in Azad College, Ausa from 1998 to 2008. He has 18 years teaching experience and his field of specialization is Nonlinear analysis, Differential and integral equations. He has supervised two students for M.Phil. and one student for Ph.D. Degree and presently guiding seven students for Ph.D. Degree. He completed one sponsored Minor research project .He is a life member of Indian Mathematical Society, Indian Science Congress, Marathwada Mathematical Society. He has published twenty two research papers in reputed National \& International Journals and also presented eleven papers in various National \& six International Conferences. He has presented seven research papers in International Conferences (Norway, Malaysia, Thailand, and India) \& ten research papers in National Conferences.

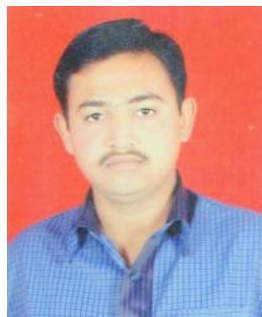

Mr. S. G. Shete, was born on 28.03.1992. He has working in the area of nonlinear differential and integral equations, functional and Random Integral equations from S. R.T.M. University Nanded from last two years. Presently he is working as Assist. Prof. Department of Mathematics, Mahatma Basweshwar Mahavidyalaya, Latur, Maharashtra. He has two years of teaching experience at Graduation and Post Graduate level and his field of specialization is Functional analysis, Nonlinear analysis, Random Integral equations. 